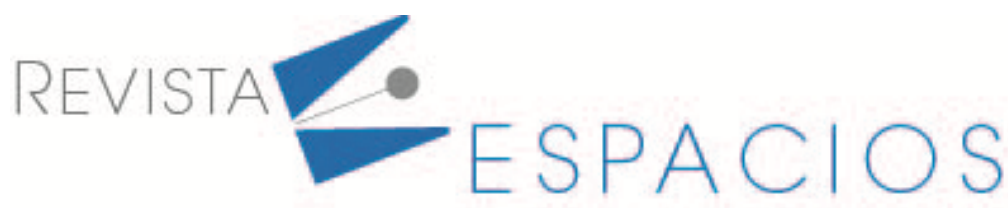

Vol. 41 (50) $2020 \cdot$ Art. 2

\title{
El inversor Dual Buck: modelado y simulación con pérdidas usando inductor simple
}

\author{
The Dual Buck inverter: loss-modeling and simulation using single inductor
}

\author{
PATARROYO-GUTIERREZ, Luis David ${ }^{1}$ \\ BAUTISTA-LÓPEZ, Diego A. ${ }^{2}$ \\ GUERRERO-BALAGUERA, Juan David ${ }^{3}$
}

\begin{abstract}
Resumen
El proceso de conversión de niveles de voltaje involucra diferentes dispositivos eléctricos y necesita varias etapas, una de ellas es la etapa de inversión, cuyo objetivo es la conversión de corriente continua a corriente alterna. El inversor es un sistema eléctrico que permite realizar la etapa de inversión. En este artículo, se estudiará el inversor Dual Buck, ya que su topología permite una alta eficiencia. Se presenta el funcionamiento y el modelo matemático del inversor utilizando dispositivos eléctricos no ideales.

Palabras clave: inversor, dbi, diagramas de bode, funciones de transferencia
\end{abstract}

\begin{abstract}
The voltage levels conversion process involves different electrical devices and needs several stages, one of them stages is the inversion stage, which it aims is the conversion from Direct-Current to Alternate-Current. Theinverterisan electrical system that allows it to perform the inversion stage. In this paper, the Dual Buck inverter will be studied, since it inverter's topology allows high efficiency. It presented the operation and mathematical model of inverter using non ideal electricdevices.

Key words: inverter, dbi, bode diagrams, transfer functions
\end{abstract}

\section{Introducción}

La necesidad de suministro energético en sitios apartados que no cuentan con conexión a la red eléctrica, es un desafío que se debe superar para lograr satisfacer las necesidades de las comunidades aisladas. Una forma de suministrar energía consiste en usar paneles fotovoltaicos, sin embargo, para entregar energía de calidad se debe contar con equipos que realicen ajustes y brinden seguridad a los usuarios en el momento de conectar electrodomésticos de uso diario. Uno de estos equipos se conoce como inversor, es el encargado de tomar la energía de los paneles fotovoltaicos que cuentan con niveles de tensión continuos y este debe convertir a niveles de tensión alterna. Por lo anterior los inversores usados deben ser confiables y eficientes en el proceso de conversión de niveles de tensión(Z. Wang, Xiao, and Yan 2006). Existen diversas configuraciones que permiten clasificar los inversores de acuerdo a niveles de tensión de entrada, elementos semiconductores y elementos de

\footnotetext{
${ }^{1}$ Docente. Facultad de Ingeniería. Escuela ingeniería Electrónica. Universidad Pedagógica y Tecnológica de Colombia. luis.patarroyo@uptc.edu.co 2Docente. Facultad de Ingeniería. Escuela ingeniería Electrónica. Universidad Pedagógica y Tecnológica de Colombia.diego.bautista03@uptc.edu.co ${ }^{3}$ Docente. Facultad de Ingeniería. Escuela ingeniería Electrónica. Universidad Pedagógica y Tecnológica de Colombia. juandavid.guerrero@uptc.edu.co
} 
filtrado. No obstante, cada configuración presenta limitaciones como la confiablidad y volumen(Hong et al. 2015), además de acuerdo a la misma se presentan problemas de recuperación inversa a través del cuerpo de los interruptores y los falsos disparos que hacen menos eficientes y confiables a los inversores(Zhou and Gao 2016). Por otra parte los cambios en el voltaje de entrada y cambios en la carga hacen que se incremente la distorsión armónica a la salida del inversor(Zhang and Song 2011). Una topología que cuenta con alta confiabilidad y eficiencia es la topología dual buck(L. Wang et al. 2016; Yao and Xiao 2012), ahora bien estos inversores usan dos inductores para el filtrado uno por cada semiciclo lo que incrementa el peso y volumen del mismo, para solucionar esto se proponen modificaciones a dicha topología utilizando únicamente un inductor(Hong et al. 2015). Para cumplir lo anterior los inversores deben ser confiables, eficientes, con un volumen reducido y adicionalmente deben controlar los niveles de tensión a la salida considerando variaciones en el voltaje de entrada(Chen et al. 2013; Yao et al. 2010).

Este artículo describe el Inversor Dual Buck (DBI) usando dispositivos eléctricos no ideales, un aporte que permite obtener la eficiencia y simular el efecto de las perdidas en el proceso de conversión de niveles de tensión, de esta forma se puede realizar una selección más adecuada de los elementos que componen el inversor en el momento de implementarlo. Se muestra el efecto de incrementar el factor de calidad del inversor y el impacto de este en la distorsión armónica en la carga. Este inversor puede ser usado en aplicaciones con paneles solares en sistemas aislados, el artículo está estructurado como sigue: en la segunda sección se presenta el DBI junto con el principio de operación, el modelo matemático con elementos no ideales se propone en la sección tres. Las funciones de transferencia que obtienen el modelo completo del inversor y los resultados de simulación que validan los análisis se muestran en las secciones cuatro y cinco. Finalmente, las conclusiones del inversor en estudio son expuestas.

\section{Metodología}

\subsection{Inversor Dual Buck de inductor simple}

El inversor DBI de inductor simple tiene las ventajas del DBI tradicional: evita el uso de tiempos muertos, no se presenta el flujo de corriente a través del cuerpo de los mosfet y se evita el problema de disparo, adicionalmente no se requieren dos inductores debido a que la configuración hace uso completo del inductor reduciendo el volumen y peso del inversor $\mathrm{DBI}$, la topologia de este circuito se muestra en la Figura 1. Esta topología es más eficiente en el sentido de que por cada semiciclo solo conmuta en alta frecuencia uno de los mosfet minimizando las pérdidas por conmutación.

Figura 1

Circuito para un inversor DBI

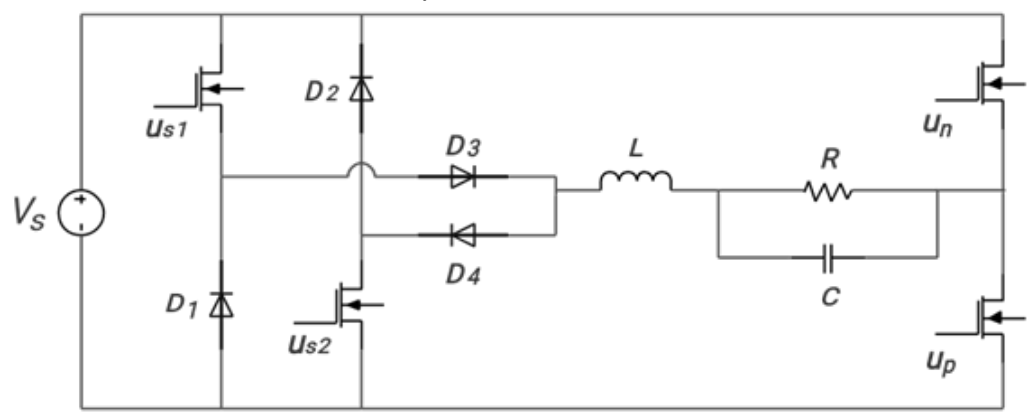

Fuente: Autores

\subsubsection{Principio de operación}

Para generar una señal senoidal a la salida del inversor se debe usar una técnica de modulación, un filtro a la salida y cuatro señales de control que definen el comportamiento del circuito. La técnica de modulación usada 
en este inversor es SPWM unipolar, debido a que a la salida del inversor presenta menos distorsión armónica reduciendo el tamaño del filtro.

Con lo establecido se determinan cuatro modos de operación definidos de acuerdo a la activación y desactivación de los mosfet del invesor. Los dos primeros modos de operación corresponden al semiciclo positivo y los siguientes al semiciclo negativo.

\section{Modo de operación 1}

En este modo de operación se entrega potencia a la carga en el semiciclo positivo, esto se logra mediante la activación de los mosfet con señales de control U_s1 y U_p, mientras los mosfet con señales de control U_s2 y U_n permanecen desactivados. El resultado de esta configuración se muestra en la figura 2.

Figura 2

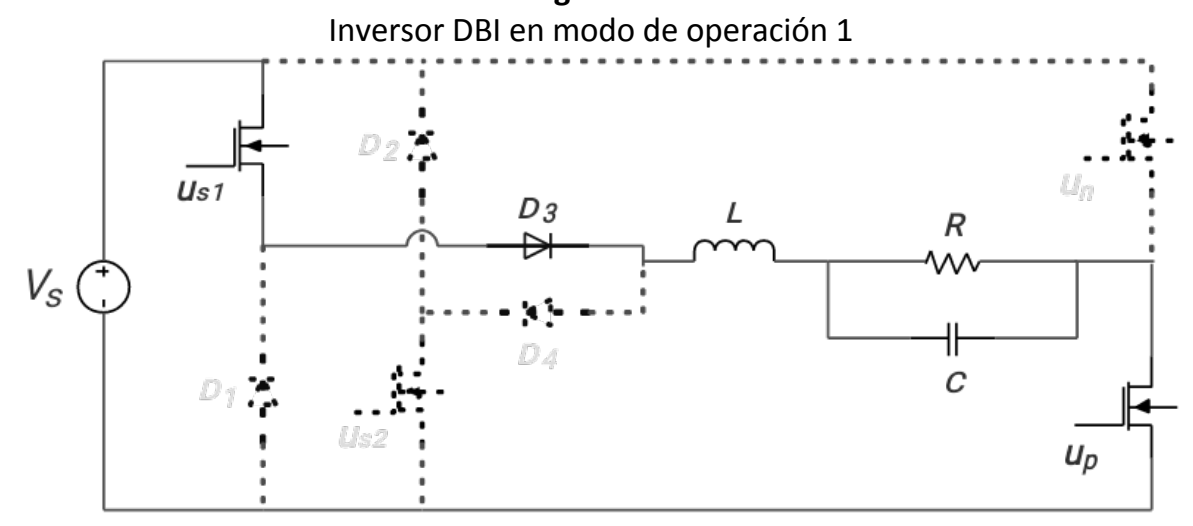

Fuente: Autores

\section{Modo de operación 2}

Durante este modo de operación, se aísla la fuente de alimentación al desactivar el mosfet con la señal de control U_s1=0, por lo que no se suministra potencia desde la fuente. La corriente fluye a través de los diodos D_1, D_2, el mosfet activado con la señal de control U_p, el filtro y la carga. Esta configuración se muestra en la figura 3.

Figura 3

Inversor DBI en modo de operación 2

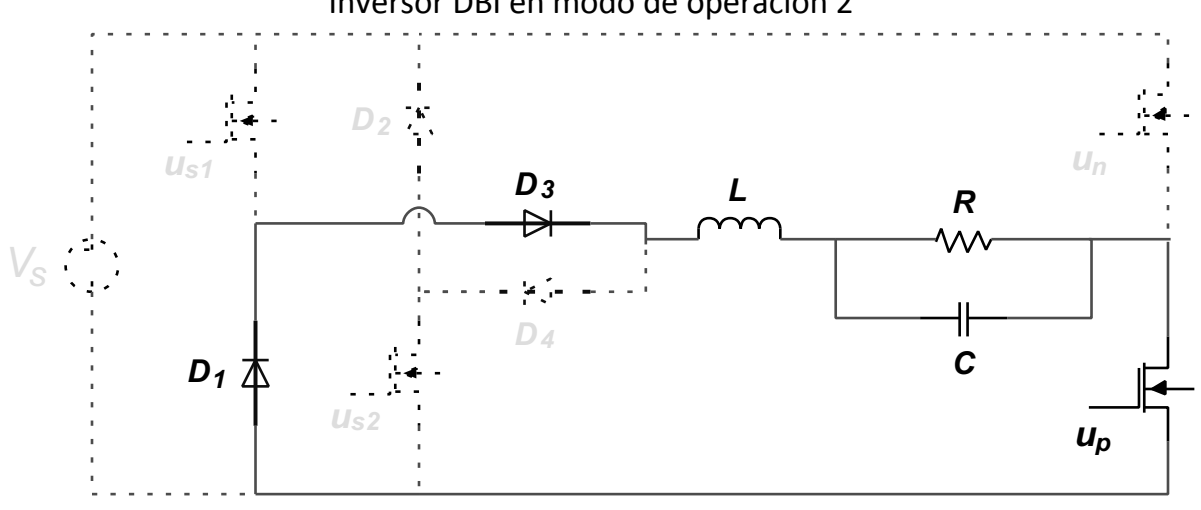

Fuente: Autores

\section{Modo de operación 3}

En este modo de operación se invierte el flujo de corriente, la configuración se establece activando los mosfet con señales de control U_s2=1 y U_n=1, los dos mosfet restantes deben estar desactivados para evitar corto circuitos. El flujo de corriente estará presente en el diodo D_4, los mosfet activados, el filtro y la carga. El circuito con esta configuración se muestra en la figura 4. 
Figura 4

Inversor DBI en modo de operación 3

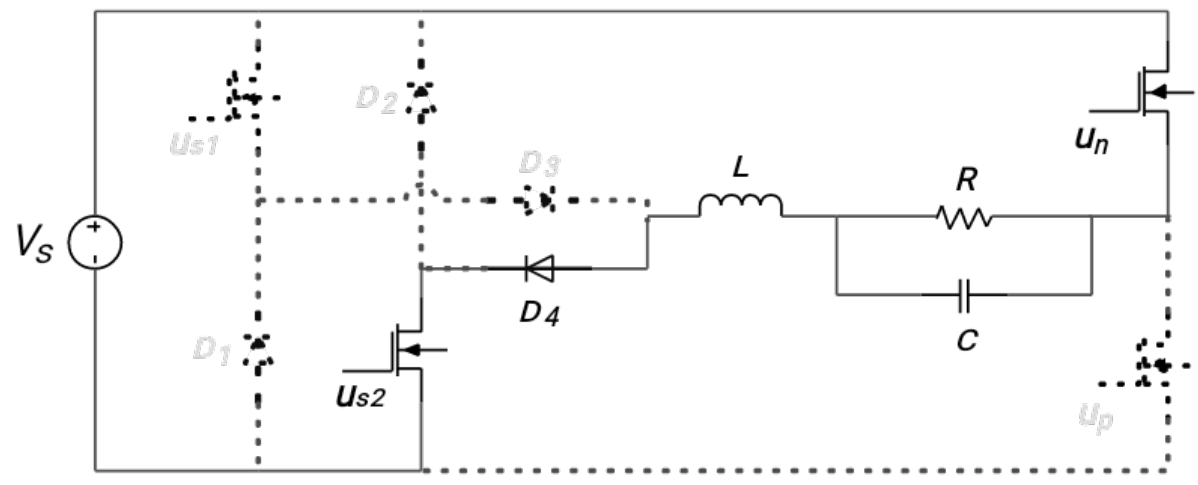

Fuente: Autores

\section{Modo de operación 4}

Para este modo de operación se aísla la fuente de alimentación al desactivar el mosfet con la señal de control U_s2 $=0$ y activado el mosfet que es controlado con la señal U_n=1. La corriente fluirá por los diodos D_2, D_4, el mosfet activado con la señal de control U_n, el filtro y la carga. Esta configuración se muestra en la figura 5.

Figura 5

Inversor DBI en modo de operación 4

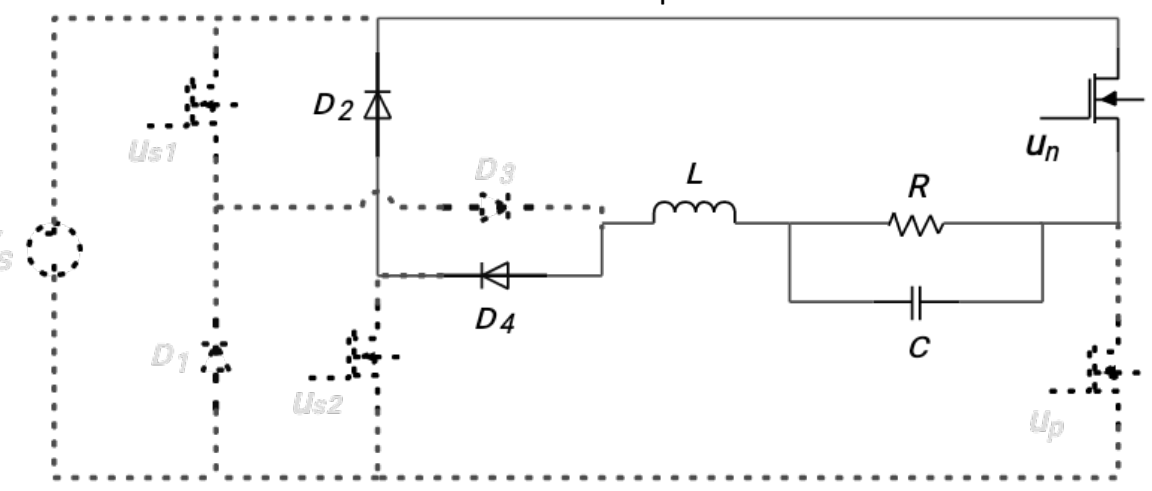

Fuente: Autores

\subsection{Modelo matemático}

Para obtener el modelo matemático del inversor se parte de la definición de los componentes reactivos en términos de corrientes y voltajes. Para este modelo se incluyen las pérdidas en los mosfet y las pérdidas en el inductor, dadas por la resistencia de encendido y la resistencia en el devanado del inductor respectivamente.

Al combinar los cuatro modos de operación y aplicando las leyes de voltaje y corriente de Kirchhoff, se definen las ecuaciones (1) y (2) que modelan la corriente en el inductor y el voltaje en el capacitor para el inversor DBI, esto de acuerdo a la conmutación de los mosfet.

$$
\begin{aligned}
& i_{L}(t)=-\frac{1}{L} R_{I N D} \cdot i_{L}(t)-\frac{1}{L} v_{c}(t)+\left[-\frac{1}{L} R_{o n} \cdot i_{L}(t)-\frac{1}{L} R_{o n} i_{L}(t) \cdot u_{s 1}(t) \cdot V_{s} \cdot u_{s 1}(t)\right] \cdot u_{3}(t) \\
& +\left[-\frac{1}{L} R_{o n} \cdot i_{L}(t)+\frac{1}{L} R_{o n} \cdot i_{L}(t) \cdot u_{s 2}(t)-\frac{1}{L} V_{s} \cdot u_{s 2}(t)\right] \cdot u_{4}(t)
\end{aligned}
$$




$$
\dot{v}_{c}(t)=\frac{1}{c} i_{L}(t)-\frac{1}{C R} v_{c}(t)
$$

Este modelo matemático permite obtener el voltaje a la salida del inversor permitiendo indentificar la eficiencia del inversor de acuerdo a los parámetros establecidos. Se llega al modelo ideal haciendo cero las resistencias de encendido de los Mosfet y la resistencia interna del inductor. En la tabla 1, se muestran los parámetros del inversor en estudio.

\section{Tabla 1}

Parámetros propuestos para el inversor DBI

\begin{tabular}{ccc} 
Parámetro & Símbolo & Valor \\
\hline Voltaje dc de entrada & $V_{S}$ & 60 \\
\hline Frecuencia de conmutación & $f_{S}$ & $50 \mathrm{kHz}$ \\
\hline Frecuencia nominal de salida & $f_{o}$ & $60 \mathrm{~Hz}$ \\
\hline Voltaje rms de salida & $v_{c}$ & $36 \mathrm{~V}$ \\
\hline Inductor & $L$ & $1 \mathrm{mH}$ \\
\hline Resistencia inductor & $R_{I N D}$ & $1 \Omega$ \\
\hline Capacitor & $C$ & $2.2 \mu \mathrm{F}$ \\
\hline Resistencia Mosfet & $R_{O n}$ & $10 \mathrm{~m} \Omega$ \\
\hline Resistor & $R$ & $23 \Omega$ \\
\hline
\end{tabular}

Fuente: Autores

\subsection{Funciones de transferencia}

En este artículo las funciones de transferencia se obtienen a partir de las ecuaciones (1) y (2), definiendo un punto de operación y realizando la linealización en el mismo. Las funciones de transferencia de la ecuación (3) relacionan el voltaje en el condensador de salida con la señales de activación de los mosfet para el semiciclo positivo y negativo respectivamente.

$$
\begin{aligned}
& H_{v \alpha_{1}}(s)=\frac{V_{s e}}{s^{2} L C+\frac{L}{R} s+1} \\
& H_{v \alpha_{2}}(s)=\frac{-V_{s e}}{s^{2} L C+\frac{L}{R} s+1}
\end{aligned}
$$

Las funciones de transferencia de la ecuación (4) permiten conocer el efecto de las variaciones del voltaje a la entrada del inversor ya que estas relacionan el voltaje en el condensador de salida con el voltaje de entrada al inversor.

$$
\begin{aligned}
& H_{v_{s p}}(s)=\frac{\alpha_{1 e}}{s^{2} L C+\frac{L}{R} s+1} \\
& H_{v_{s n}}(s)=\frac{-\alpha_{2 e}}{s^{2} L C+\frac{L}{R} s+1}
\end{aligned}
$$


En la figura 6 se muestra el diagrama de bloques de las funciones de transferencia que definen el modelo completo de la planta(Bacha, Munteanu, and Bratcu 2014), esto se logra al aplicar el principio de superposición lineal considerando las variaciones en las señales de control y las variaciones en la tensión de entrada al sistema.

Con las funciones de transferencia y aplicando el principio de superposición lineal se obtiene el modelo completo de la planta como se muestra en la figura 6.

Figura 6

Inversor DBI en lazo abierto definido a

partir de funciones de transferecia

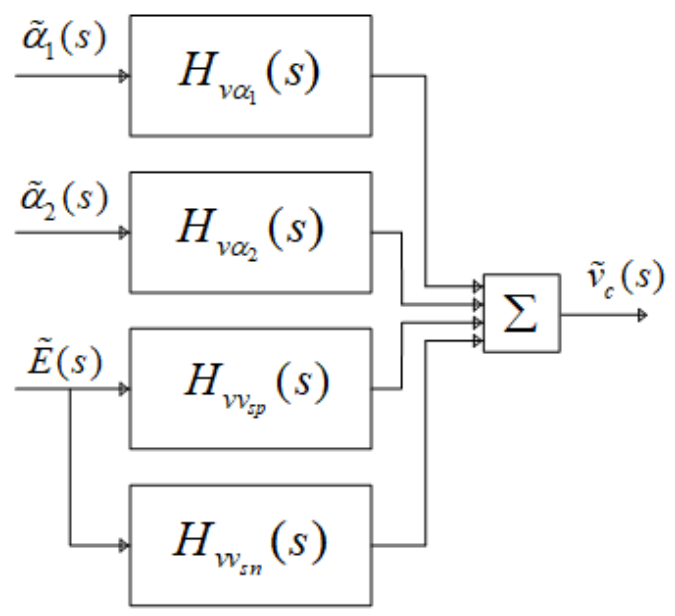

Fuente: Autores

\section{Resultados}

Para generar la señal seniodal se utiliza la modulación SPWM unipolar que se obtiene a partir de la comparación de una señal triangular y una señal seniodal(Su et al. 2015). Como resultado de la modulación se tienen cuatro señales que permiten realizar la conmutación de los mosfet como se indica en el principio de operación del DBI. Estas señales de control son las que ingresan a los bloques de las funciones de transferencia de la figura 6 . La respuesta del sistema se obtiene al aplicar el principio de superposición con las funciones de transferencia dadas en las ecuaciones (3) y (4). La figura 7 muestra el voltaje de salida del inversor en un punto de operación según parámetros de diseño considerando la modulación SPWM como entrada en las funciones de transferencia de la figura 6.

Figura 7

Voltaje en la carga en el punto de operación 1

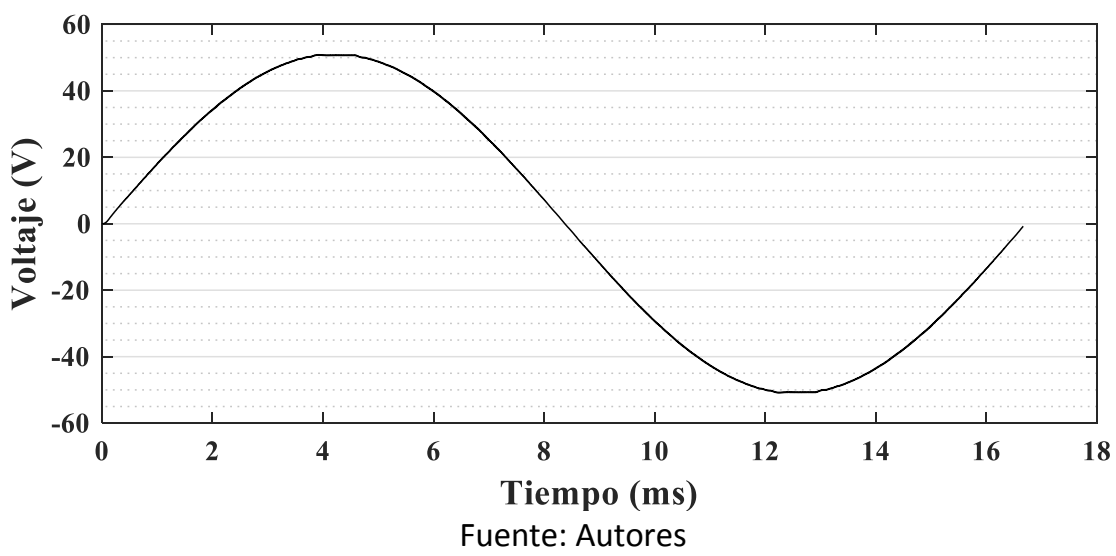


En la figura 8 se muestra la respuesta del sistema en un punto de operación con una carga 20 veces mayor, luego el factor de calidad Q es alto incrementando la distorsión armónica de la señal de salida, se presenta distorsión en la señal cerca a los picos de la señal y en los curces por cero.

Figura 8

Voltaje en la carga en el punto de operación 2

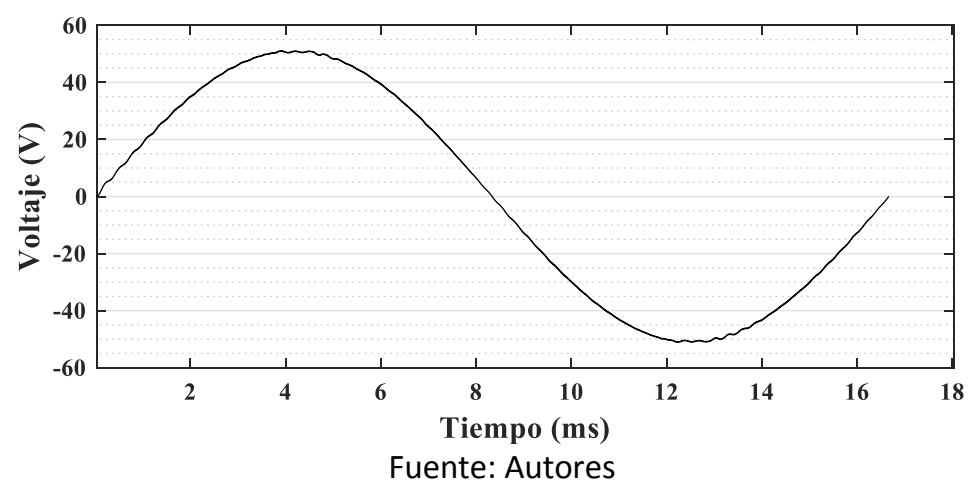

La respuesta en frecuencia del inversor para tres factores $Q$ se presenta en la figura 9, este diagrama de bode representa la respuesta de la función de transferencia Gvd, el mayor sobre pico y el cambio brusco en la fase indica que el parámetro $Q$ es alto. A medida que se incrementa la impedancia en la carga se incrementa $Q$ (Rashid 2004). El DBI con los parámetros definidos se muestra en la misma figura con un factor de calidad bajo por lo que no se presentan sobre picos ni cambios bruscos en la fase, logrando una distorsión armónica baja en el voltaje de salida del inversor.

Figura 9

Diagrama de Bode DBI para la función de transferencia Grd con tres puntos de operación

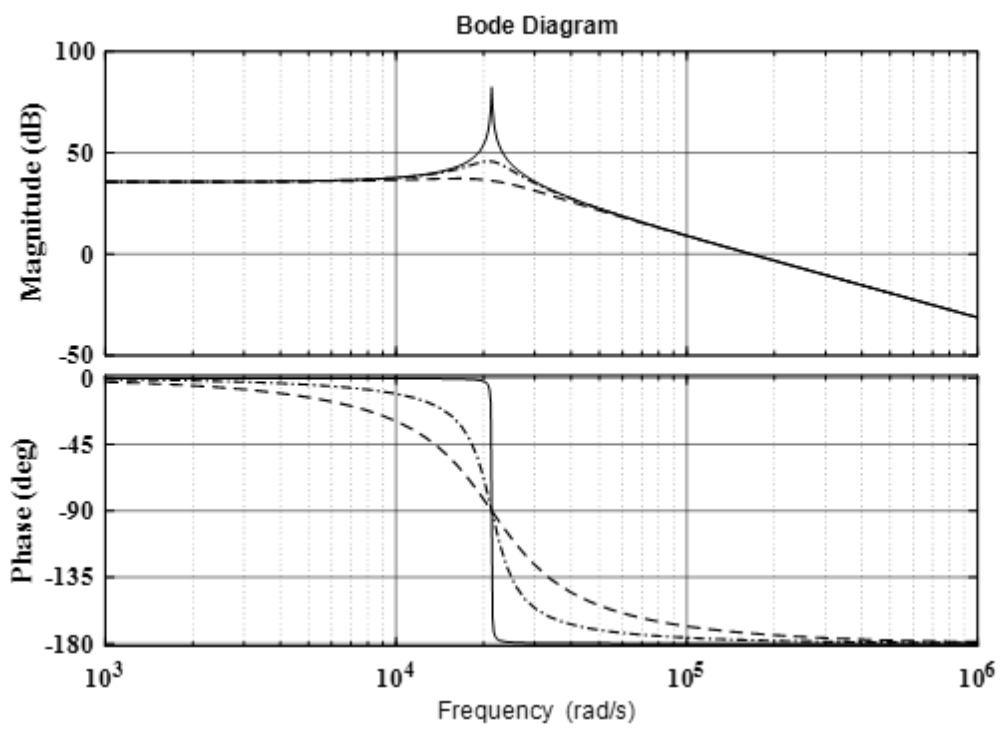

Fuente: Autores

\section{Conclusiones}

Se ha presentado el modelo del Inversor Dual Buck con elementos no ideales a partir de la conmutación dada por el principio de operación. 
La variación de la carga conectada al inversor hace que cambie el punto de operación del mismo y con esto el factor de calidad, modificando la respuesta en frecuencia al presentarse sobre picos y cambios rápidos en la fase junto con oscilaciones en la respuesta en el tiempo a medida que se incrementa el factor de calidad.

De acuerdo al modelo propuesto se requieren cuatro señales de control, dos para el semiciclo positivo y dos para el semiciclo negativo para controlar el voltaje de salida con una modulación SPWM unipolar, por cada semiciclo uno de los transistores opera en baja frecuencia lo que reduce las pérdidas de conmutación al implementar el inversor.

\section{Referencias bibliográficas}

Bacha, Seddik, IulianMunteanu, and AntonetalulianaBratcu. 2014. "Power Electronic Converters Modeling and Control", Advanced Textbooks in Control and Signal Processing. Springer London. http://dx.doi.org/10.1007/978-1-4471-5478-5.

Chen, Baifeng et al. 2013. "Current Distortion Correction in Dual Buck Photovoltaic Inverter with a Novel PWM Modulation and Control Method." Conference Proceedings - IEEE Applied Power Electronics Conference and Exposition - APEC: 727-31.

Hong, Feng et al. 2015. "Single Inductor Dual Buck Full-Bridge Inverter." IEEE Transactionson Industrial Electronics 62(8): 4869-77.

Rashid, Muhammad H. 2004. "Electrónica de Potencia", Circuitos, Dispositivos y Aplicaciones. Pearson Educación.

Su, Tong et al. 2015. "A High Power Density Dual-Buck Full-Bridge Inverter Based on Carrier Phase-Shifted SPWM Control." : 1715-21.

Wang, Lu, Yan Li, Qi Yan, and Wei Dou. 2017. “Dual Buck Grid-Connected Inverter Based on GaN Devices." 2016 Asian Conference on Energy, Power and Transportation Electrification, ACEPT 2016 (2014).

Wang, Zan, Lan Xiao, and Gguang Yan. 2006. "Simulation Study of Charge Controlled Half-Cycle Modulated Dual Buck Half Bridge Inverter." PESC Record - IEEE Annual Power Electronics Specialists Conference (1).

Yao, Zhilei, and Lan Xiao. 2012. "Two-Switch Dual-Buck Grid-Connected Inverter with Hysteresis Current Control." IEEE Transactions on Power Electronics 27(7): 3310-18.

Yao, Zhilei, Lan Xiao, Xing Wei, and Huizhen Wang. 2010. "Dual-Buck Full-Bridge Inverter with SPWM Control and Single Current Sensor." Proceedings of the 2010 5th IEEE Conference on Industrial Electronics and Applications, ICIEA 2010 1: 2154-58.

Zhang, Xianjin, and Yongxian Song. 2011. "A Control Method of Dual Buck Half Bridge Inverter Based on the Phase of Voltage Loop Output." Proceedings - 3rd International Conference on Measuring Technology and Mechatronics Automation, ICMTMA 2011 1(2): 25-27.

Zhou, Liwei, and Feng Gao. 2016. "Dual Buck Inverter with Series Connected Diodes and Single Inductor." Conference Proceedings - IEEE Applied Power Electronics Conference and Exposition - APEC 2016-May(c): 2259-63. 